\title{
Infrared laser-based monitoring of the silane dissociation during deposition of silicon thin films
}

\author{
R. Bartlome, ${ }^{\text {a) }}$ A. Feltrin, and C. Ballif \\ Ecole Polytechnique Fédérale de Lausanne (EPFL), Institute of Microengineering (IMT), \\ Photovoltaics and Thin Film Electronics Laboratory, Rue A.-L. Breguet 2, 2000 Neuchâtel, Switzerland
}

(Received 25 March 2009; accepted 3 May 2009; published online 21 May 2009)

\begin{abstract}
The silane dissociation efficiency, or depletion fraction, is an important plasma parameter by means of which the film growth rate and the amorphous-to-microcrystalline silicon transition regime can be monitored in situ. In this letter we implement a homebuilt quantum cascade laser-based absorption spectrometer to measure the silane dissociation efficiency in an industrial plasma-enhanced chemical vapor deposition system. This infrared laser-based diagnostic technique is compact, sensitive, and nonintrusive. Its resolution is good enough to resolve Doppler-broadened rotovibrational absorption lines of silane. The latter feature various absorption strengths, thereby enabling depletion measurements over a wide range of process conditions. (C) 2009 American Institute of Physics. [DOI: 10.1063/1.3141520]
\end{abstract}

One of the current major challenges of the thin-film silicon photovoltaic industry is the deposition of hydrogenated microcrystalline silicon $(\mu \mathrm{c}-\mathrm{Si}: \mathrm{H})$ in large-area industrial plasma-enhanced chemical vapor deposition (PECVD) systems. A crucial parameter for high-rate deposition of devicegrade $\mu \mathrm{c}-\mathrm{Si}: \mathrm{H}$ is the silane depletion fraction. In a recent study, ${ }^{1}$ it has been shown that the growth of $\mu \mathrm{c}-\mathrm{Si}: \mathrm{H}$ occurs at low silane concentrations in the plasma, i.e., when the input $\mathrm{SiH}_{4}$ flow is sufficiently diluted in $\mathrm{H}_{2}$ or when the silane is sufficiently depleted within the plasma. In the latter case, device-grade $\mu \mathrm{c}-\mathrm{Si}: \mathrm{H}$ can even be grown from a pure $\mathrm{SiH}_{4}$ flow. ${ }^{2}$ In addition to investigations of the phase transition from amorphous silicon to microcrystalline silicon, silane depletion measurements enable-in the absence of polysilane dust particles- the monitoring of the film growth rate, as shown below. Another method to determine in situ the amorphous-to-microcrystalline transition is to monitor the $\mathrm{SiH}_{4}$ density during a short postdeposition $\mathrm{H}_{2}$ plasma etch. ${ }^{3}$

Prior attempts to directly measure the depletion fraction of silane were based upon mass spectrometers, ${ }^{4-6}$ and a Fourier transform infrared spectrometer (FTIR) featuring a typical resolution of $0.5 \mathrm{~cm}^{-1}{ }^{1,7}$ While the former are bulky and intrusive, the latter lacks of sensitivity. ${ }^{8}$ Recently, optical emission spectroscopy has proven to be a compact and inexpensive technique to measure the depletion fraction in hydrogen-diluted plasmas, ${ }^{8}$ but such measurements require the assumption of a plasma model in which the electronic temperature must remain constant. This is typically not fulfilled in dusty plasmas or plasmas with very high silane concentrations. ${ }^{8}$ In this letter, we propose to measure the silane depletion fraction under any plasma condition using a tunable IR laser spectrometer.

Silane molecules feature intense rotovibrational absorption lines between 4.35 and $4.76 \mu \mathrm{m} .{ }^{9,10}$ Unfortunately, this is also a mid-IR range that is not easily accessible with current laser sources. Noncryogenic-cooled tunable mid-IR laser sources in this wavelength range were basically limited to complex systems such as optical parametric oscillators and difference frequency generation sources. ${ }^{11}$ Nowadays, with

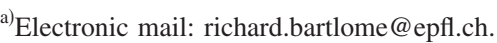

the advent of the room-temperature continuous-wave quantum cascade laser (QCL) ${ }^{12}$ numerous opportunities are arising in the field of gas analysis. ${ }^{13-15}$ In this letter, we operate a Peltier-cooled continuous-wave distributed-feedback QCL (Alpes Lasers SA, Switzerland). ${ }^{16}$ Its single-mode emission is tunable between 2241 and $2245 \mathrm{~cm}^{-1}$. This wavenumber range is particularly well suited for silane depletion measurements, as it is free from any known interferences with other absorbing species in the atmosphere. The QCL is mounted in a compact housing of the size of a cigar box. By varying the current supplied to the QCL, the wavenumber is tuned with a slope of $-21 \mathrm{~cm}^{-1} / \mathrm{A}$. The highly divergent QCL beam is collimated by a telescope consisting of a custom-made $\mathrm{Ge}$ aspheric lens and $\mathrm{a} \mathrm{CaF}_{2}$ planoconvex lens. The mid-IR beam is modulated with a mechanical chopper at a frequency of $1 \mathrm{kHz}$. Before mounting the spectrometer on the PECVD chamber, the beam quality and collimation were controlled using a $\mathrm{Hg}-\mathrm{Cd}-\mathrm{Te}$ camera. Two $\mathrm{Ag}$ mirrors guide the mid-IR beam through a $3.7 \mathrm{~m}$ long evacuation line. The windows of the evacuation line are wedged to avoid the effect of overlapping multiple reflections. The mid-IR beam exiting the evacuation line is focused on a thermoelectric-cooled $\mathrm{Hg}$ $\mathrm{Cd}-\mathrm{Te}$ photovoltaic detector. The preamplified detector signal is read by a computer-controlled lock-in amplifier, the reference signal of which is provided by the chopper controller. The whole optical setup is pictured in Fig. 1.

Depletion measurements were taken in the evacuation line of an industrial KAI-M PlasmaBox ${ }^{\mathrm{TM}}$ reactor, ${ }^{17}$ the total inner surface of which was about $0.616 \mathrm{~m}^{2}$. The interelectrode gap was $13 \mathrm{~mm}$. Hydrogen and silane were introduced in the reactor through a uniform showerhead built in the

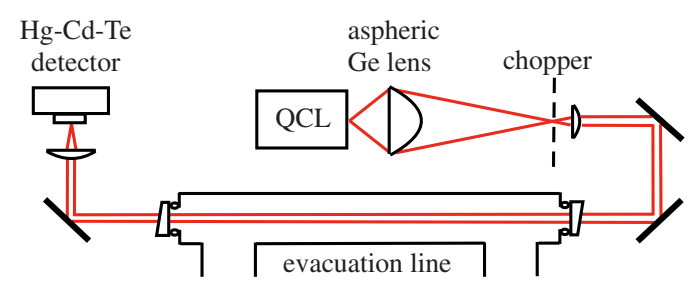

FIG. 1. (Color online) Optical setup of the QCL-based IR spectrometer. 


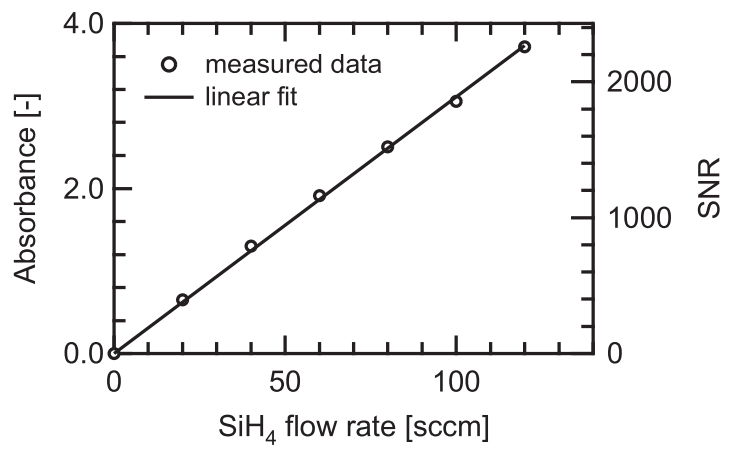

FIG. 2. Silane absorbance and SNR at $2243.83 \mathrm{~cm}^{-1}$ vs the input $\mathrm{SiH}_{4}$ flow rate before igniting the plasma. The input $\mathrm{H}_{2}$ flow rate and the total pressure in the PECVD reactor were maintained at 700 SCCM and 4.5 mbars, respectively.

electrode. Exhaust gases were pumped from one side. A butterfly valve regulated the pressure inside the reactor. Thin $\mathrm{Si}$ films were deposited at a constant substrate temperature of $453 \mathrm{~K}$ and at a very high excitation frequency (VHF) of $40.68 \mathrm{MHz}$.

The input $\mathrm{SiH}_{4}$ in a PECVD reactor can either contribute to the film growth, can polymerize to form powderlike particles, ${ }^{18}$ or may not react at all and be pumped out of the reactor. Before ignition of the hydrogen-diluted plasma, the input $\mathrm{SiH}_{4}$ mass flow rate $\dot{m}_{\mathrm{SiH}_{4}}$ is completely pumped through the evacuation line. If $\dot{m}_{\mathrm{SiH}_{4}}^{\text {of }}$ is the $\mathrm{SiH}_{4}$ mass flow rate in the evacuation line before ignition of the plasma, we can write $\dot{m}_{\mathrm{SiH}_{4}}^{\text {off }}=\dot{m}_{\mathrm{SiH}_{4}}$. After ignition of the plasma, the $\mathrm{SiH}_{4}$ mass flow rate in the evacuation line $\dot{m}_{\mathrm{SiH}_{4}}^{\mathrm{on}}$ can be expressed as

$$
\dot{m}_{\mathrm{SiH}_{4}}^{\text {on }}=(1-D) \dot{m}_{\mathrm{SiH}_{4}}^{\text {off }},
$$

where $D$ is the silane dissociation efficiency. If a beam of light and a constant flow of absorbing species are passed through a vacuum line, the mass flow rate $\dot{m}$ is given by

$$
\dot{m}=\rho \cdot S \cdot \nu=\frac{M}{N_{A}} \cdot n \cdot S \cdot \nu=\frac{M}{N_{A}} \cdot \frac{A}{\sigma L} \cdot S \cdot \nu,
$$

where $\rho$ is the mass density, $S$ is the section of the line, $\nu$ is the velocity, $M$ is the molar mass, $N_{A}$ is the Avogadro number, $n$ is the number density, $A$ is the absorbance defined in terms of the natural logarithm in the Beer-Lambert law, $\sigma$ is the absorption cross section, and $L$ is the absorption path length. In general, absorption cross sections depend on the wavelength, the total pressure, and the temperature. For small pressures below 10 mbars, as found in PECVD, the rotovibrational absorption lines of $\mathrm{SiH}_{4}$ are Dopplerbroadened. Their shape can be described by a Gaussian function that does not depend on the total pressure anymore. Preignition measurements, pictured in Fig. 2, together with Eq. (2), show that the $\mathrm{SiH}_{4}$ number density in our evacuation line is proportional to the input $\mathrm{SiH}_{4}$ mass flow rate. Therefore, with Eqs. (1) and (2), the dissociation efficiency can be expressed as

$$
D=1-\frac{\dot{m}_{\mathrm{SiH}_{4}}^{\mathrm{on}}}{\dot{m}_{\mathrm{SiH}_{4}}^{\mathrm{off}}}=1-\frac{A_{\mathrm{SiH}_{4}}^{\mathrm{on}}}{A_{\mathrm{SiH}_{4}}^{\mathrm{off}},}
$$

where $A_{\mathrm{SiH}_{4}}^{\mathrm{on}}$ and $A_{\mathrm{SiH}_{4}}^{\mathrm{off}}$ are the absorbances in the evacuation line measured at a given wavenumber before and after ignition of the plasma, respectively. In literature, ${ }^{1,7}$ the right hand side of Eq. (3) formally defines the depletion fraction, whereas the dissociation efficiency is defined by Eq. (1). In this letter, both expressions are found to be equivalent, in agreement with Ref. 5.

In the absence of polysilane particles, the silicon deposition rate $\dot{m}_{\mathrm{Si}}^{\text {dep }}$ is given by

$$
\dot{m}_{\mathrm{Si}}^{\mathrm{dep}}=D \cdot \dot{m}_{\mathrm{SiH}_{4}} \cdot \frac{M_{\mathrm{Si}}}{M_{\mathrm{SiH}_{4}}}
$$

where $M_{\mathrm{SiH}_{4}}$ and $M_{\mathrm{Si}}$ are the molar masses of silane and silicon, respectively. The silicon deposition rate $\dot{m}_{\mathrm{Si}}^{\mathrm{dep}}$, expressed in units of $\mathrm{kg} / \mathrm{s}$, is related to the film growth rate $R_{g}$,

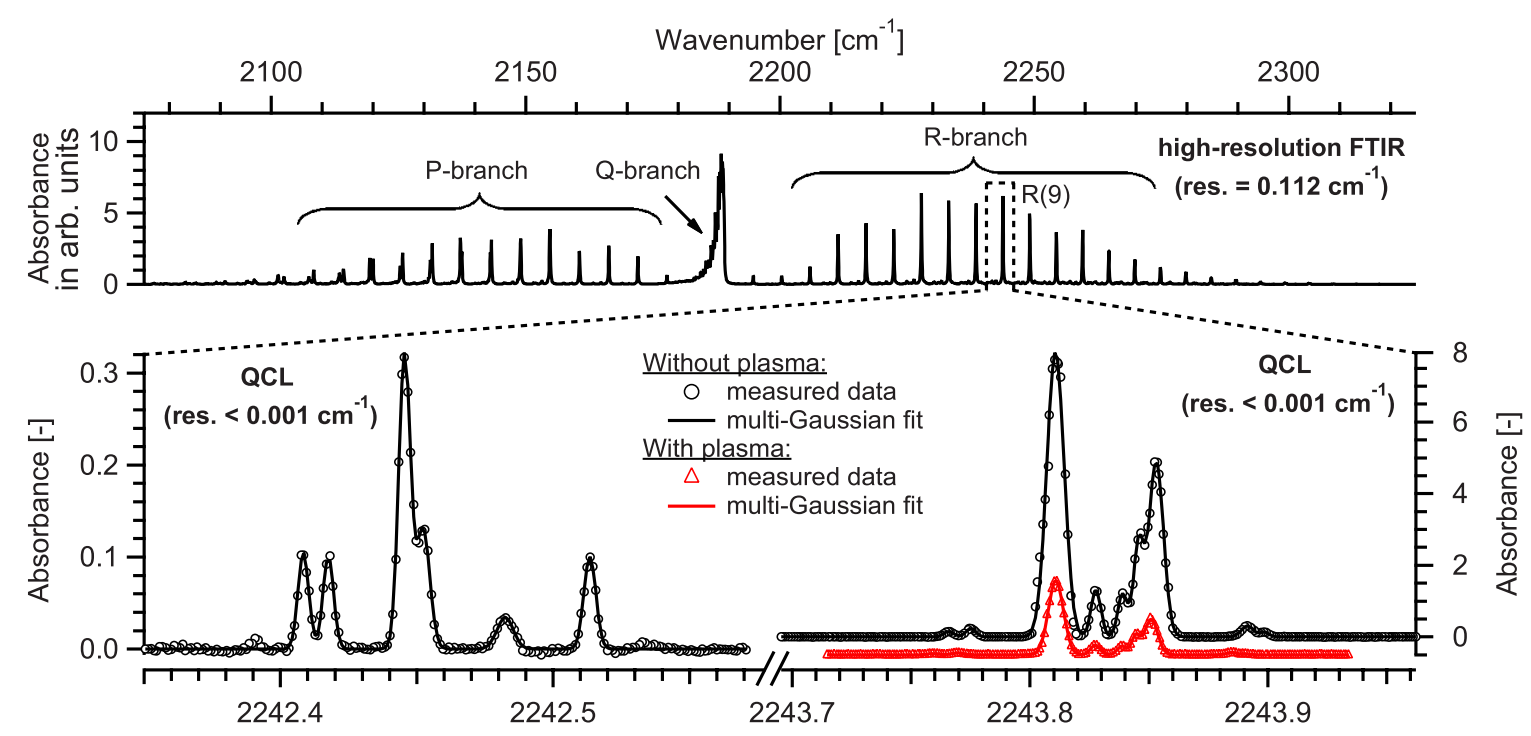

FIG. 3. (Color online) The $\nu_{3}$ band of $\mathrm{SiH}_{4}$ acquired by a high-resolution FTIR (upper part of the figure; see Ref. 10), the Doppler-resolved R(9) multiplet of $\mathrm{SiH}_{4}$ acquired by the QCL before and after igniting the plasma in the high-deposition-rate regime (right hand side of the inset), and weak unassigned absorption lines of $\mathrm{SiH}_{4}$ that only appear in the spectrum acquired by the QCL (left hand side of the inset). For illustrative purposes, the spectrum of $\mathrm{SiH}_{4}$ acquired after igniting the plasma is slightly shifted in the vertical axis. 
expressed in units of $\mathrm{m} / \mathrm{s}$, through the following equation:

$$
\dot{m}_{\mathrm{Si}}^{\mathrm{dep}}=R_{g} \cdot A_{\text {react }} \cdot \rho_{\mathrm{Si}},
$$

where $A_{\text {react }}$ is the inner surface of the reactor and $\rho_{\mathrm{Si}}$ is the silicon mass density of the film. Given the low atomic percentage of $\mathrm{H}_{2}$ in such films, $\rho_{\mathrm{Si}}$ can be approximated by the mass density of the film itself $\rho_{\mathrm{Si}: \mathrm{H}}$, which is $2.2-2.3 \mathrm{~g} / \mathrm{cm}^{3}$ for $\mu \mathrm{c}-\mathrm{Si}: \mathrm{H}$ thin films. ${ }^{19,20}$ The growth rate $R_{g}$ can be determined by comparing Eqs. (4) and (5),

$$
R_{g}=\frac{D \cdot \dot{m}_{\mathrm{SiH}_{4}} \cdot \frac{M_{\mathrm{Si}}}{M_{\mathrm{SiH}_{4}}}}{A_{\text {react }} \cdot \rho_{\mathrm{Si}}} .
$$

During preliminary measurements, the QCL was calibrated with an accuracy of $0.01 \mathrm{~cm}^{-1}$ with the help of the well-documented $\mathrm{N}_{2} \mathrm{O} \nu_{3}$-band rotational lines $\mathrm{R}(23), \mathrm{R}(24)$, $R(25)$, and $R(26)$. As pictured in Fig. 3, silane spectra were then acquired by scanning the injection current of the QCL with steps of $50 \mu \mathrm{A}$ at a rate of $1.7 \mathrm{~s}$ per step. Given the step width, the precision of the spectrometer is better than $0.001 \mathrm{~cm}^{-1}$ (the linewidth of the laser emission itself is much lower). The QCL-based spectrum of $\mathrm{SiH}_{4}$ features nine absorption lines that have been assigned to the R(9) multiplet of the $\nu_{3}$ vibrational band. ${ }^{21}$ These lines are centered at $2243.766, \quad 2243.775, \quad 2243.810, \quad 2243.827, \quad 2243.839$, 2243.847, 2243.852, 2243.891, and $2243.898 \mathrm{~cm}^{-1}$. Furthermore, six unassigned weak absorption lines of $\mathrm{SiH}_{4}$ appear at $2242.408,2242.418,2242.445,2242.453,2242.483$, and $2242.514 \mathrm{~cm}^{-1}$. The peak centered at $2243.810 \mathrm{~cm}^{-1}$ is saturated in Fig. 3 and not suited for quantitative measurements under our process conditions. By comparing the peak centered at $2243.827 \mathrm{~cm}^{-1}$ under steady-state conditions before and after ignition of the plasma, depletion fractions of $22.4 \pm 1.3 \%$ and $80.0 \pm 1.3 \%$ were measured when $\mu \mathrm{c}-\mathrm{Si}: \mathrm{H}$ films were deposited at a low-deposition rate and at a highdeposition rate, respectively. In the low-deposition-rate regime, the total pressure in the reactor was maintained at 3.5 mbars, the upper electrode was powered at $0.05 \mathrm{~W} / \mathrm{cm}^{2}$, and the input $\mathrm{SiH}_{4}$ and $\mathrm{H}_{2}$ flow rates were 50 and $2000 \mathrm{SCCM}$ (SCCM denotes cubic centimeter per minute at STP), respectively. In good agreement with an ex situ profilometric measurement on the film thickness that yielded a growth rate of $1.53 \pm 0.03 \AA / \mathrm{s}$, a growth rate of $1.7 \pm 0.1 \AA / \mathrm{s}$ is derived from the depletion measurement and Eq. (6). In the highdeposition-rate regime, the total pressure in the reactor was maintained at $4.5 \mathrm{mbars}$, the VHF power was $0.12 \mathrm{~W} / \mathrm{cm}^{2}$, and the input $\mathrm{SiH}_{4}$ and $\mathrm{H}_{2}$ flow rates were 40 and 700 SCCM, respectively. In good agreement with an ex situ profilometric measurement on the film thickness that yielded a growth rate of $4.37 \pm 0.17 \AA / \mathrm{s}$, a growth rate of
$4.8 \pm 0.2 \AA / \mathrm{s}$ is derived from the depletion measurement and Eq. (6). Nonuniform deposition regimes would account for larger discrepancies between in situ and ex situ measurements.

In conclusion, the silane dissociation efficiency was measured in this letter with an unprecedented level of precision using an IR laser spectrometer. This diagnostic technique is compact, sensitive, and nonintrusive. It was mounted on an industrial PECVD system for in situ monitoring of the film growth rate. Doppler-resolved rotovibrational absorption lines of $\mathrm{SiH}_{4}$ have been resolved. Given their various absorption strengths and the excellent signal-to-noise ratio (SNR) of the spectrometer, depletions measurements over a wide range of process conditions are feasible.

The authors acknowledge helpful discussions with B. Strahm, J.-L. Kumin, and S. De Wolf.

${ }^{1}$ B. Strahm, A. A. Howling, L. Sansonnens, and C. Hollenstein, Plasma Sources Sci. Technol. 16, 80 (2007).

${ }^{2}$ M. N. van den Donker, B. Rech, F. Finger, W. M. M. Kessels, and M. C. M. van de Sanden, Appl. Phys. Lett. 87, 263503 (2005).

${ }^{3}$ G. Dingemans, M. N. van den Donker, A. Gordijn, W. M. M. Kessels, and M. C. M. van de Sanden, Appl. Phys. Lett. 91, 161902 (2007).

${ }^{4}$ J. R. Doyle, D. A. Doughty, and A. Gallagher, J. Appl. Phys. 68, 4375 (1990).

${ }^{5}$ M. N. van den Donker, B. Rech, W. M. M. Kessels, and M. C. M. van de Sanden, New J. Phys. 9, 280 (2007).

${ }^{6}$ S. Nunomura, I. Yoshida, and M. Kondo, Appl. Phys. Lett. 94, 071502 (2009).

${ }^{7}$ L. Sansonnens, A. A. Howling, and C. Hollenstein, Plasma Sources Sci. Technol. 7, 114 (1998).

${ }^{8}$ A. A. Howling, B. Strahm, P. Colsters, L. Sansonnens, and C. Hollenstein, Plasma Sources Sci. Technol. 16, 679 (2007).

${ }^{9}$ P. Chollet, G. Guelachvili, M. Morillon-Chapey, P. Gressier, and J. P. M. Schmitt, J. Opt. Soc. Am. B 3, 687 (1986).

${ }^{10}$ S. W. Sharpe, T. J. Johnson, R. L. Sams, P. M. Chu, G. C. Rhoderick, and P. A. Johnson, Appl. Spectrosc. 58, 1452 (2004).

${ }^{11}$ M. W. Sigrist, R. Bartlome, D. Marinov, J. M. Rey, D. E. Vogler, and H. Wachter, Appl. Phys. B: Lasers Opt. 90, 289 (2008).

${ }^{12}$ M. Beck, D. Hofstetter, T. Aellen, J. Faist, U. Oesterle, M. Ilegems, E. Gini, and H. Melchior, Science 295, 301 (2002).

${ }^{13}$ G. Duxbury, N. Langford, M. T. McCulloch, and S. Wright, Chem. Soc. Rev. 34, 921 (2005).

${ }^{14}$ A. Kosterev, G. Wysocki, Y. Bakhirkin, S. So, R. Lewicki, M. Fraser, F. Tittel, and R. F. Curl, Appl. Phys. B: Lasers Opt. 90, 165 (2008).

${ }^{15}$ J. Ropcke, S. Welzel, N. Lang, F. Hempel, L. Gatilova, O. Guaitella, A. Rousseau, and P. B. Davies, Appl. Phys. B: Lasers Opt. 92, 335 (2008).

${ }^{16}$ T. Aellen, S. Blaser, M. Beck, D. Hofstetter, J. Faist, and E. Gini, Appl. Phys. Lett. 83, 1929 (2003).

${ }^{17}$ J. Perrin, J. Schmitt, C. Hollenstein, A. Howling, and L. Sansonnens, Plasma Phys. Controlled Fusion 42, B353 (2000).

${ }^{18}$ C. Hollenstein, Plasma Phys. Controlled Fusion 42, R93 (2000).

${ }^{19}$ Z. Remes, M. Vanecek, P. Torres, U. Kroll, A. H. Mahan, and R. S Crandall, J. Non-Cryst. Solids 227, 876 (1998).

${ }^{20}$ J. Mullerova, P. Sutta, G. van Elzakker, M. Zeman, and M. Mikula, Appl. Surf. Sci. 254, 3690 (2008).

${ }^{21}$ A. Cabana, L. Lambert, and C. Pepin, J. Mol. Spectrosc. 43, 429 (1972). 